\title{
Tangence
}

\section{Effets de filtre en lecture littéraire}

\section{Christian Vandendorpe}

Numéro 36, mai 1992

La lecture littéraire

URI : https://id.erudit.org/iderudit/025708ar

DOI : https://doi.org/10.7202/025708ar

Aller au sommaire du numéro

Éditeur(s)

Tangence

ISSN

0226-9554 (imprimé)

1710-0305 (numérique)

Découvrir la revue

Citer cet article

Vandendorpe, C. (1992). Effets de filtre en lecture littéraire. Tangence, (36), 19-33. https://doi.org/10.7202/025708ar d'utilisation que vous pouvez consulter en ligne.

https://apropos.erudit.org/fr/usagers/politique-dutilisation/ 


\section{Effets de filtre en lecture littéraire}

\section{Christian Vandendorpe}

Longtemps considérée comme pure et simple activité de décodage entièrement "programmée" par l'auteur (ou le texte), la lecture apparaît de plus en plus comme une activité polymorphe et difficile à cerner, dont la complexité est à rattacher à la richesse des processus que nous mettons en jeu pour comprendre le monde.

Nous n'examinerons pas ici les difficultés que suscite la lecture d'un texte informatif: même si elle peut paraître peu problématique, on a montré que celle-ci est nécessairement orientée en fonction des besoins d'information du lecteur et de ses connaissances ${ }^{1}$.

Ce qui est vrai du texte informatif doit l'être encore plus du texte littéraire, car ce "comportement finalisé" qu'est la lecture ${ }^{2}$ n'est même pas contraint et encadré par la nécessité de repérer et de recueillir un contenu objectif traduisible en termes de connaissances ou d'informations.

Mais existe-t-il une lecture proprement littéraire? J'entends par là non pas une "lecture des textes littéraires" mais une "lecture littéraire des textes ". Poser une telle question revient à transporter dans le domaine de la réception l'interrogation que l'on pose depuis Jakobson sur la littérarité des textes ${ }^{3}$, et qui a fait l'objet de synthèses récentes par Mircéa Marghescou, Thomas Aron, Yves Reuter et Marc Angenot, sans oublier l'ouvrage collectif de Louise Milot et Fernand Roy ${ }^{4}$.

1 Michel Pagé et Gilles Primeau, *Critères généraux pour évaluer l'habileté à lirex, Québec français, n 46, mai 1982, p. 60-64.

2 Frans Rutten, "Sur les notions de texte et de lecture dans une théorie de la réception*, Revue des sciences bumaines, $\mathrm{n}^{\circ}$ 177, janvier-mars 1980, p. $67-83$.

3 Le terme literaturnost a été introduit dans une conférence en 1919. Mais il n'apparaîtra en français qu'avec Tzvetan Todorov, Théorie de la littérature, Paris, Seuil, 1965, p. 37.

4. Thomas Aron, Littérature et littérarité, Paris, Les Belles lettres, 1984; Mircéa Marghescou, Le concept de littérarité, Paris, Mouton, 1974; Yves Reuter, •Définir les biens littéraires *, Pratiques, 67, septembre 1990, p. 514; Marc Angenot, * Frontières des études littéraires: science de la 
Si l'on excepte un regain d'intérêt au cours des dernières années, il faut reconnaître que cette recherche avait généralement été abandonnée parce qu'elle ne semblait pas pouvoir donner de résultat sûr. Plus on l'examine, en effet, plus le littéraire s'apparente à un objet flou, rebelle à la catégorisation. Selon les termes d'Yves Reuter, la frontière en est essentiellement "poreuse" et il semble que "sa spécificité serait sa non-spécificité ". L'impossibilité d'assigner des bornes au littéraire a en effet été montrée à maintes reprises. Borges nous a habitués à des fictions où se mêlent extraits d'encyclopédie et d'ouvrages de philosophie. Et l'on sait depuis Breton qu'il est possible d'intégrer dans une œuvre poétique un extrait d'annuaire téléphonique, cas extrême s'il en est 5 ! Inversement, il est possible de lire un poème de façon à en évacuer toute poésie, et l'effet humoristique d'une telle transgression est d'autant plus fort que celle-ci s'applique à une œuvre connue et réputée (voir Julos Beaucarne lisant "Le lac" de Lamartine).

Tout cela confirme qu'il existe bien une façon "littéraire" de lire, qui va tendre à produire des effets similaires dans un public donné et déclencher une expérience commune. C'est en jouant sur cette dimension esthétique des textes (contenue implicitement dans l'adjectif "littéraire") qu'une société va aussi pouvoir valoriser tel texte plutôt que tel autre. À partir de cette constatation que la littérature joue un "rôle socialisateur", on peut certes l'envisager comme un phénomène social - ce qu'elle est assurément -, et les sociologues ont brillamment éclairé cette dimension. Mais comme toute pratique sociale, cet babitus ${ }^{6}$ qu'est la lecture littéraire se manifeste aussi, chez l'individu, par un fonctionnement cognitif particulier, et c'est ce dernier que je voudrais examiner ici pour tâcher d'en identifier, de façon forcément schématique et exploratoire, certaines caractéristiques.

Mon hypothèse est que, considéré sous l'angle de la réception, le "littéraire" peut être redéfini comme un filtre formé d'un

littérature, science des discours *, Horizons philosopbiques, vol. $1, \mathrm{n}^{\circ} 1$, p. 23-34; Louise Milot et Fernand Roy (sous la direction de), La littérarité, Québec, Presses de l'Université Laval, 1991.

5 André Breton, Clair de terre, Paris, Gallimard, 1966.

6 L'habitus désigne un savoir implicite, emmagasiné de façon inconsciente et automatique au cours de pratiques signifiantes. Ce concept est utilisé par Pierre Bourdieu, :Disposition esthétique et compétence artistique., Les temps modernes, 1971, n 295, p. 1345-1378. Voir aussi La Distinction, Paris, Minuit, 1979. 
ensemble de représentations appliquées à des textes et de prescriptions sur la façon de les lire 7 . Cette image, qui évoque une lentille utilisée en photographie ou un dispositif électro-acoustique de filtrage des sons, met en évidence le fait qu'une bonne part de nos opérations mentales se font "à travers " ou "à partir" de quelque chose, particulièrement celles qui impliquent un jugement. Il ne faudrait cependant pas se laisser abuser par le côté concret de cette image et céder à son côté quelque peu fixiste. Nos constructions mentales, en effet, sont éminemment souples, et les recherches psychologiques ont montré que nos expériences tendent à se réorganiser spontanément sous des formes plus abstraites, grâce auxquelles nous sommes à même d'effectuer des généralisations et des prédictions ${ }^{8}$. Le savoir que nous accumulons, au fil de nos expériences, au sujet d'une réalité quelconque n'est pas emmagasiné de façon inerte dans notre mémoire, mais passe à travers différents circuits cérébraux (néo-cortex, hippocampe, lobe temporal...) pour déboucher sur la formation de réseaux d'information hiérarchisés ${ }^{9}$, parmi lesquels on identifie notamment le schéma et le "prototype". Ce dernier définit une classe d'objets en renvoyant à un représentant éminent de la classe, tout en laissant la possibilité à d'autres objets de se distinguer de ce dernier par certaines de leurs propriétés. C'est ainsi que l'image du chien est susceptible de jaillir en réponse au mot "animal "bien plus souvent et avec plus de spontanéité que celle du protozoaire ou de l'ornithorynque (dont une recherche plus approfondie nous convaincra pourtant qu'ils appartiennent bien à la même classe).

7 Le concept de filtre que je propose ici partage certains traits avec celui de régime utilisé par Marghescou, op. cit., et qui est d'inspiration phénoménologique. Dans les deux cas, il s'agit d'une instance propre à l'acte de Iecture et qui mobilise une posture cognitive susceptible de modifications.

8 Ce phénomène a été mis en évidence de manière expërimentale par F. Bartlett, Remembering, Cambridge, Cambridge University Press, 1932. Pour une présentation synthétique des recherches sur le schéma, consulter le chapitre 2 de mon livre Apprendre à lire des fables. Une approcbe sémiocognitive, Longueuil, Le Préambule/Balzac, coll. "L'univers des discours *, 1989.

9 Cette réorganisation inconsciente de nos informations en mémoire, qui avait été montrée par Bartlett, op.cit, et explorée au cours des années soixante-dix dans les recherches en intelligence artificielle (Schank, Abelson, Minsky), a été récemment mise en évidence dans le fonctionnement des circuits anatomiques du cerveau (voir notamment Larry Squire, Memory and Brain, New York, Oxford University Press, 1987). 
De la même façon peut-on supposer que nous nous formons un "prototype" du littéraire de plus en plus englobant à mesure que s'étend notre expérience de la littérature. C'est ce qui nous permet de reconnaître les textes susceptibles d'être lus à partir de lui, et d'en éprouver l'adéquation à une intention personnelle de plaisir esthétique.

\section{L'expérience originelle de la littérature}

Si l'on accepte de définir minimalement la lecture littéraire comme un état de plaisir esthétique provoqué par le langage (en retrouvant ainsi le Barthes du Plaisir du texte), on devra admettre que cet état ne constitue pas un palier auquel on accéderait soudainement à un moment déterminé de notre existence et dont la découverte constituerait une expérience à laquelle rien ne nous aurait préparés. En fait, il suffit d'observer des enfants pour constater qu'ils tirent jouissance du langage dès leur plus jeune âge. Encore au berceau, l'enfant s'amuse à répéter en écho, explore les sonorités, se laisse bercer par des rimes, des comptines. Il expérimente alors ce qui constitue sans doute le premier niveau du littéraire, la poésie dominée par le "signifiant-roi", laquelle occupe une place originelle dans toutes les littératures. Â peu près en même temps, l'enfant découvre une autre composante de base du littéraire, qui va probablement l'accompagner une bonne partie de sa vie: il accède par l'enchantement des récits à la dimension fondamentale de la narrativité et aux innombrables jeux qu'elle permet. Un peu plus tard, il découvre ou pressent que ces histoires merveilleuses, contes de fées, fables, récits de toute sorte, ne lui parlent pas seulement de personnages lointains mais lui apportent des aperçus nouveaux sur lui-même, et les symboles qu'il y trouve vont se mettre à flamboyer comme autant d'étoiles dans son univers intérieur: la lecture lui permet alors de "S'inventer", selon la belle expression de Suzanne Lamy ${ }^{10}$. Si, à ces trois dimensions, on ajoute la prise de conscience des jeux que permet le fonctionnement métaphorique (ce mécanisme de base du langage et que la poésie exploite de façon privilégiée), on possède alors le socle cognitif des jugements de littérarité, sur lequel l'école et la société vont tendre, par la suite, à imposer leurs propres constructions. 


\section{Mouvance du littéraire}

Il y a autre chose à l'œuvre, aussi, dans le jugement de littérarité, que cette expérience personnelle du langage et de la lecture. Pour s'en convaincre, il n'est que de se rappeler que la lecture littéraire ne privilégie pas les mêmes objets d'une époque à l'autre.

Parmi les exemples qu'on peut évoquer à cette fin, il suffit de considérer le phénomène représenté par l'abbé Jacques Delille (1738-1813). Auteur de poèmes descriptifs et didactiques, ce dernier était tenu en son temps comme l'égal de Virgile, dont il avait d'ailleurs fait une traduction réputée (Voltaire lui-même l'appelait "Virgilius Delille»). Son succès fut immense et a inspiré nombre d'imitateurs. On raconte qu'émigré à Londres, sa femme le forçait à rimer ses trente vers chaque matin, qui êtaient ensuite monnayés au prix fort. À sa mort, il eut droit à des funérailles grandioses qui ne furent égalées que par celles de Victor Hugo. Or, depuis, ce "prince des poètes" est aussi méprisé qu'il a pu être louangé. Flaubert, dit-on, en avait fait sa lecture favorite lorsqu'il était en humeur de bouffonner. Et, pour Alain, Delille est le représentant typique de "la poésie sans la poésie "11. Pourtant, c'est à lui qu'Édouard Guitton rapporte l'épanouissement de la poésie descriptive en France entre 1760 et 1800 , dont il était le chef de file incontesté ${ }^{12}$. Mais cette poésie qui délaie ses descriptions sous des périphrases pompeuses, ne trouve plus guère qu'une poignée de défenseurs (et à peine plus de lecteurs) dans le public d'aujourd'hui. Il semble que se soit évanoui le goût qu'on avait alors pour découvrir la description d'une locomotive dans les vers

Surtout quand de Papin l'hermétique clôture

Concentre dans l'airain la chaleur qu'il endure

ou pour décrypter le nom du cochon sous «l'animal qui se nourrit de glands"!

Force est donc d'imputer la qualité "littéraire" de cette poésie à la présence d'un filtre particulier chez les lecteurs, qui s'attachait à des caractéristiques de l'œuvre jugées importantes à l'époque alors qu'elles ne le sont plus aujourd'hui. Ainsi faut-il supposer que la reconnaissance d'un procédé considéré alors comme littéraire, telle la périphrase, entraînait chez le lecteur un jugement de littérarité

11 Cité par Georges Maurevert, Le livre des plagiats, A. Fayard, Paris, 1922.

12 Édouard Guitton, Jacques Delille et le poème de la nature en France de 1750 à 1820, Paris, Klincksieck, 1974. 
24

— tout comme la découverte de segments rimés déclenche encore facilement dans le grand public un jugement de poésie. Mais, dans le public lettré d'aujourd'hui, cette attention aux figures ne semble plus faire partie intégrante de la lecture littéraire et l'on a disqualifié toute tentative de faire coïncider la littérature avec la mise en œuvre de figures données. Le filtre méta-rhétorique est tout simplement considéré comme non pertinent parce que tombé en désuétude. Ainsi que l'a noté Genette, ala fonction auto-signifiante de la Littérature ne passe plus par le code des figures, et la littérature moderne a sa propre rhétorique, qui est précisément (pour l'instant du moins) le refus de la rhétorique "13. On est certes encore capable de reconnaître le procédé et d'appréhender l'œuvre où il apparaît comme relevant du domaine littéraire, mais on ne tire plus guère de gratification de sa lecture. Tout se passe comme avec un cliché: on reconnaît dans le procédé l'intention potentiellement littéraire de l'utilisateur naiff et un écho affaibli de son effet originel, mais il ne déclenche plus rien.

La littérarité d'un procédé n'est donc pas une constante mais une variable, qui est établie par la critique en conjonction avec l'esprit du temps et l'exigence de renouvellement inhérente à la création d'objets esthétiques.

Peut-être faudrait-il rappeler qu'il n'y a pas de compartiments étanches entre littérature et critique et que l'un et l'autre sont étroitement imbriqués, l'écriture prenant fond souvent sur une réflexion critique de premier ordre. Avant de se lancer dans la création, Proust a répondu à Sainte-Beuve et longuement réfléchi sur les procédés d'écriture des écrivains qu'il aimait, afin de s'en approprier le style. Un des jugements les plus cinglants qu'il ait proférés porte sur la faible détermination stylistique des comparaisons chez Flaubert: réflexion capitale, qui éclaire en contrepartie l'extrême soin qu'il apporte lui-même à ses métaphores et la façon dont il les développe ${ }^{14}$.

À la limite, l'écrivain voudra être jugé très précisément à l'intérieur d'un paradigme littéraire donné. On en trouve un exemple déjà ancien chez Chapelain (1595-1694), qui contribua à établir les canons du classicisme et qui, dans la préface de $L a$ Pucelle, "supplie ceux qui verront ce travail que, quand il en

13 Gérard Genette, Figures I, Paris, Seuil, coll. *Points *, 1966, p. 221.

14 Marcel Proust, Chroniques, Paris, Gallimard, 1949. 
feront l'examen, ils s'examinent les premiers, et sachent bien auparavant s'ils possèdent les lumières nécessaires pour prononcer sur son Invention, sur sa Disposition et sur son Élocution" 15. Voilà quelqu'un qui était tellement conscient du rôle que joue un filtre déterminé dans le jugement de littérarité qu'il aurait aimé restreindre la lecture de son œuvre à ceux qui partageaient les mêmes préconstruits que lui !

De la même façon, toute avant-garde n'a de cesse que d'avoir publié son manifeste, livre-repère où sont énoncées les normes d'écriture selon lesquelles elle prétendra renouveler la littérature et être jugée par ses lecteurs. On pense, bien sûr, au Manifeste du surréalisme de Breton, à Pour un nouveau roman de RobbeGrillet, ou à Refus global de Borduas. Mais il faudrait y ajouter toutes les œuvres qui ont été enfantées parce qu'elles répondaient à un paradigme en émergence. Ainsi, par exemple, Tournier notet-il que Zola aurait écrit Le roman expérimental en réponse à L'introduction à l'étude de la médecine expérimentale de Claude Bernard, afin d'établir que "la littérature sera scientifique ou ne sera pas" "16. Qu'on pense aussi à Sartre lisant Mauriac et cherchant à imposer un code narratif où l'auteur ne pourrait plus se dissimuler sous un narrateur omniscient.

Le créateur est donc à la merci de la pensée critique en vigueur dans une société donnée. Il peut chercher à s'y soustraire en imposant son propre canon. Mais, le plus souvent., il va créer à l'intérieur des contraintes existantes, quitte à revendiquer son affiliation à une chapelle déterminée, car il a toujours besoin d'un minimum de lecteurs témoins. Ou encore, il va utiliser les méthodes mêmes de la critique pour construire son ouvre. Italo Calvino a ainsi dialogué avec les sémioticiens, en montrant que son roman Si par une nuit d'biver un voyageur repose sur une architecture rigoureuse de quarante-deux carrés sémiotiques dont chacun produit six axiomes, par simple application du parcours canonique, et que ces carrés sont à leur tour répartis en douze chapitres, selon une distribution de type pyramidal ${ }^{17}$.

15 Jean Suberville, Théorie de l'art et des genres littéraires, Paris, Éd. de l'École, 1955, p. 11-12.

16 Michel Tournier, Le nouvel observateur, $n^{\circ} 1406,17-23$ octobre 1991, p. 6.

17 Italo Calvino, - Comment j'ai écrit un de mes livres, Actes sémiotiques (Documents), Centre national de la recherche scientifique, vol. Vl, n ${ }^{\circ} 51$, 1984. 
Il est donc abusif et mystificateur d'opposer radicalement critique et création, comme a tenté de le faire le romantisme. En fait, selon la formule de Louise Milot, discours critique et littérature constituent "deux sujets d'énonciation sur le seul terrain qui leur soit à tous deux pertinent "18. C'est d'ailleurs dans cet échange dynamique entre littêrature et commentaire qu'Yves Reuter fait reposer la littérarité ${ }^{19}$.

\section{Le filtre idéologique}

Le filtre cognitif dont il a été question jusqu'ici, et qui subsume la totalité de nos expériences relatives à un même champ, est en principe, ainsi qu'on l'a vu, une construction purement automatique. L'activité inconsciente dont il procède peut toutefois être modifiée par suite d'une intervention délibérée sur nos contenus d'expériences et d'une réinterprétation de celles-ci en fonction d'un axiome superstructurant: c'est le fonctionnement idéologique.

Marc Angenot a récemment réfuté l'idée althussérienne selon laquelle l'idéologie serait un système et rappelé que tout énoncé peut être qualifié d'idéologique ${ }^{20}$. Pour ma part, je retiendrai ce dernier terme afin de désigner un type particulier de fonctionnement mental. Un énoncé est idéologique quand il est imposé comme instance de décodage ultime par suite de notre appartenance ou de notre soumission à un groupe, à un parti, voire à une société ou à une religion. En ce sens, l'idéologie fonctionne comme un opérateur cognitif qui, appliqué à une réalité quelconque, tend à donner toujours les mêmes résultats. Elle constitue une forme de pensée socialisée qui vise à tisser entre les membres d'un groupe une parfaite docilité aux mots d'ordre, en décourageant toute interprétation personnelle de la réalité. Elle peut renvoyer à un énonciateur explicite, qui agira alors comme argument d'autorité ultime, ou au contraire camoufler son origine et se donner comme une émanation "spontanée" de la collectivité. Dans les deux cas, l'idéologie cherche à s'imposer comme LE contexte obligé de tout énoncé. À la différence de la coutume

18 Louise Milot, * La figure de l'écrit: son inscription dans le texte et dans l'histoire littéraire , in Louise Milot et Femand Roy, op. cit., p. 9.

19 "[...] le bien littéraire n'est ni le texte, ni le commentaire [...] mais le rapport texte-commentaire, lui-même intégré dans un champ spécifique et des institutions qui lui sont liées*, Reuter, loc. cit., p. 9-10.

20 Marc Angenot, "L'idéologie n'est pas un système *, CIADEST, 1991. 
ou des conventions sociales, l'idéologie existe d'abord sous la forme d'un contenu discursif: c'est ce qui en fait la grande "portabilité "à travers les classes sociales et les cultures. Elle est un contenu axiomatique qui n'a pas besoin d'être démontré, parce que la démonstration est censée en avoir été faite ailleurs ou en d'autres temps. Ce qui différencie l'idéologie, comme contexte cognitif, de nos autres schèmes de pensée, c'est à la fois son fort degré de socialisation et le niveau auquel elle opère, l'ampleur du champ qu'elle couvre. S'appuyant sur l'effet de vérité qu'elle projette, elle vise à saturer le champ du discours et à se poser en instance ultime de légitimation de toute action sociale.

Dans les cas les moins favorables, le filtre idéologique appliqué à une œuvre littéraire peut avoir pour effet d'évincer son objet, ou de larges pans de celui-ci, voire de le nier tout simplement. Les dernières décennies ayant été particulièrement riches à cet égard, elles recèlent quantité d'exemples de ce phénomène. Nous n'en examinerons qu'un seul, qui a trait à la définition même du littéraire. Dans leur Introduction aux Français fictifs, Balibar et Macherey posent le principe que "l'effet esthétique est toujours aussi un effet de domination: assujettissement des individus à l'idéologie dominante, et domination de l'idéologie de la classe dominante " 21 et visent à conclure que cet effet est inculqué par l'école. Mais comment rendre compte, dans cette perspective, du caractère transhistorique de la culture et du fait que des œuvres anciennes puissent encore nous toucher aujourd'hui? Voyons comment nos auteurs escamotent la question:

On lève par là-même, au sein de la tradition marxiste, les énigmes d'un problème insoluble, un problème qui tout simplement n'existe pas comme tel: celui que semblait poser Marx à la fin de l'Introduction de 1857 à la Contribution à la critique de l'Économie politique lorsqu'il se demandait comment l'"art " d'une époque révolue, tout entier relatif aux rapports sociaux, et en particulier aux rapports idéologiques de son temps, pouvait posséder encore pour les hommes de la société bourgeoise un "charme" indestructible, apparemment éternel. Mais le "charme de l'art grec" n'est pas du tout éternel, pour la bonne raison que, chez les Grecs, il n'était pas celui d'un art; l'lliade n'était pas une "cuure littéraire", ni les tragédies de Sophocle, pas plus que l'architecture du Parthénon ou les statues de Praxitèle

21 Renée Balibar, Les français fictifs, présentation d'Étienne Balibar et de Pierre Macherey, Paris, Hachette, 1974, p. 46. 
n'étaient des " œuvres d'art ". C'est rétrospectivement que l'iliade ou Gidipe Roi ont été transformés en textes "littéraires" et ont pu produire des effets littéraires au sein d'une formation idéologique nouvelle. (p. 24-25, nous soulignons)

Ainsi, le fameux problème que Marx "semblait poser " n'existerait tout simplement pas "comme tel"! Il suffit, pour cela, par un habile saupoudrage de guillemets, de restreindre la notion de littérature aux productions de la société bourgeoise, et de rejeter aux oubliettes l'héritage antérieur, ce qui permet à nos auteurs de sauvegarder l'intégrité de leur propre idéologie en s'imaginant que rien n'échappe à son système logico-déductif... Beau cas de sociologisme vulgaire, où les conditions sociales qui gouvernent l'exercice de la production littéraire sont seules prises en considération, indépendamment du matériau sur lequel cette production s'exerce et des effets qu'elle entraîne chez les lecteurs/auditeurs.

Certes, il n'est pas question de nier que ces conditions sociales aient radicalement changé au $\mathrm{XIX}^{\mathrm{e}}$ siècle, où l'on assiste à une professionnalisation de la littérature. Mais il ne faudrait pas confondre cet appareil externe avec les caractéristiques internes (et les exigences) de la production littéraire. À cet égard, le témoignage de Proust, qui avait réfuté par anticipation une telle position, nous semble plus conforme à la réalité de l'expérience esthétique:

Que les conditions extérieures de la production littéraire aient changé au cours du dernier siècle, que le métier d'homme de lettres soit devenu chose plus absorbante et exclusive, c'est possible. Mais les lois intérieures, mentales, de cette production n'ont pas pu changer. ${ }^{22}$

$A$ fortion Proust aurait-il rejeté l'idée que les lois intérieures de la réception de l'œuvre littéraire aient pu changer et que l'épopée homérique ait pu ne pas apparaître comme une ceuvre d'art pour les anciens Grecs!

Pour comprendre que l'on puisse encore aujourd'hui frémir à la lecture de l'Iliade, il faut probablement raffiner le modèle sociologique par la prise en compte du fonctionnement cognitif. Si l'on admet que notre jugement de littérarité se forme au contact répété d'œuvres caractérisées comme littéraires, on doit logiquement s'attendre à ce que celui-ci se développe à la façon d'un prototype,

22 Marcel Proust, Contre Sainte-Beuve, Paris, Gallimard, coll. * Folio *, 1954, p. 219-220. 
incorporant les traits les plus généraux des ouvres lues et hiérarchisant leurs caractéristiques secondaires en raison de leur fréquence d'occurrences et de leur position dans le champ culturel. C'est ce filtre qui nous permet d'apprécier un texte non encore médiatisé, voire anonyme, ou de goûter une œuvre aussi éloignée de nous par le temps et l'espace que peut l'être, par exemple, le Genji Monogatari de Murasaki Shikibu (Japon, 1004). Plus le filtre est raffiné, plus s'élargit cette capacité, dans un mouvement potentiellement infini. Et l'on peut faire l'hypothèse que l'œuvre dite "classique" est celle qui combine le plus d'éléments placés très haut dans la hiérarchie du prototype littéraire, ce qui explique que ce type d'œuvre soit spontanément évoqué pour rendre compte des productions dérivées d'une même esthétique.

\section{Le filtre littéraire aujourd'hui}

Certes, il se produit des modifications considérables dans l'appréciation du littéraire et celles-ci peuvent affecter des segments plus ou moins importants de notre héritage, comme on l'a vu avec l'infortuné Delille. L'histoire de la littérature est remplie de ces réévaluations et toute relecture d'une œuvre passée est susceptible d'en modifier la signification. Comme l'écrit Robert Major, "une ceuvre qui traverse le temps devient forcément polysémique, entrant dans des séries culturelles différentes, jouant des rôles différents pour différents lecteurs" 23 .

En fait, en examinant le livre des entrées et sorties au palmarès de la littérarité, on pourrait voir se dessiner cette "Histoire de l'esprit " que Valéry appelait de ses voux ${ }^{24}$. Certains genres ont perdu presque tout halo de littérarité. C'est le cas de la fable, notamment, qui, sans avoir jamais été un genre élevé, a joui d'un statut assez respectable pour tenter un poète de premier plan comme La Fontaine et qui, dans les trois derniers quarts du XVIII siècle, a même été considérée comme un genre littéraire à part entière $^{25}$. On sait le médiocre statut qu'elle possède aujourd'hui. D'autres textes ont gagné en littérarité. Il n'est que de songer au

23 Robert Major, Jean Rivard ou l'art de réussir, Québec, Presses de l'Université Laval, 1991, p. 14.

24 Paul Valéry, Cabiers, 2 tomes, Paris, Gallimard, La Pléiade, 1957.

25 Thomas Noël, Theories of the Fable in the Eigbteentb Century, New York, Columbia University Press, 1975, p. 4. 
30

destin de la poésie hermétique, avec Mallarmé, en comparaison avec le sort de ses contemporains tels Leconte de Lisle ou Sully Prudhomme (prix Nobel).

De toute évidence, le filtre littéraire actuel est placé sous le signe du "sens étoilé" (Barthes), du maillage infini des connotations. Il se caractérise par une dévalorisation du limpide au profit de la polysémie. Le sens ultime doit rester autant que possible indécidable. Et c'est cette indécidabilité qui est de plus en plus censée produire chez le lecteur l'effet de littérarité. C'est en cela, très précisément, que je peux tomber d'accord avec la thèse de Todorov selon laquelle le genre fantastique serait "la quintessence de la littérature * ${ }^{26}$ et qu'il serait aujourd'hui omniprésent ${ }^{27}$.

George Steiner rattache l'établissement de ce nouveau paradigme à Mallarmé et à Rimbaud, et parle à ce propos de "crise du sens" 28 . J'ai montré ailleurs qu'il s'agit plutôt de "l'ouverture d'un nouveau registre dans le fonctionnement du texte " 29 et que l'écrivain d'aujourd'hui déconstruit les automatismes linguistiques les plus subtils pour produire dans l'esprit du lecteur l'impression de décollage du réel caractéristique de la littérature.

Ce nouveau paradigme a entraîné une relecture et une réévaluation des œuvres du passé, visant à mettre en évidence ce qu'elles recèlent d'ambigu et de polysémique. La Fontaine résiste encore assez bien à une telle opération parce qu'il a beaucoup joué sur la prosodie et la polyphonie afin de nuancer ce que les fables classiques pouvaient avoir de schématique et de trop limpide. Mais la lecture des fables n'a pas toujours mis en évidence cette polysémie. Ainsi, Hegel reprochait au contraire à la fable sa limpidité: "Elle est comme une énigme qui serait toujours accompagnée de sa solution. "30 On peut apprécier le renversement de

26 Tzvetan Todorov, Introduction à la littérature fantastique, Paris, Seuil, 1970, p. 176. C'était évidemment avant que le même auteur ne conclue à la non-existence de la littérature.

27 Pour une discussion de la thèse de Todorov sur le fantastique, voir mon article intitulé .Pouvoirs du héros et rationalité dans le fantastique et la science-fiction, à paraître dans Actes du colloque sur les rapports entre le fantastique et la science-fiction, Université Laval, 1992.

28 George Steiner, Réelles présences. Les arts du sens, Paris, Gallimard, 1991.

29 . Contexte, compréhension et littérarité *, $R S / S I$, vol. XI, $\mathrm{n}^{\circ} 1,1991$, p. 21.

30 Georg Wilhelm Hegel, Esthétique, textes choisis par Claude Khodoss, Paris, PUF, 1962, p. 175. 
paradigme quand on lit aujourd'hui dans un dictionnaire que "La fable joue de cette équivoque sur le sens. Elle induit le lecteur vers des solutions équiprobables. [...] Sa polysémie la rend proprement insensée. 31

Tout se passe comme si l'utilisation univoque du langage était frappée de nullité littéraire et irrémédiablement rejetée du côté de la parole journalistique ou scientifique. Barthes a sans doute été le premier à énoncer ce nouveau paradigme dans son $S / Z$, où il prend position contre "les philologues, décrétant que tout texte est univoque, détenteur d'un sens vrai, canonique" (p. 13) et où il revendique au contraire une lecture foisonnante, plurielle.

Pourtant, il semble bien que la littérature, dans ses œuvres archétypales, ait toujours joué sur un au-delà du sens. Mais, longtemps, cet au-delà du sens a été donné, voire tenu pour garanti, par la métrique. En faisant jouer à la fois le plan du signifié et l'ordre du musical, le poème se dérobe à une appréhension univoque et oblige à faire intervenir le non-sens absolu qu'est le pur signifiant dans la constitution du sens, tâche en définitive impossible et qui laisse toujours inachevé le commentaire. Aujourd'hui que la métrique est sortie de la littérature, sans doute sous la poussée d'une civilisation davantage axée sur le visuel que sur le phonique, on a récupéré sous une autre forme ce trait tenu pour essentiel au texte littéraire et qui est l'impossibilité d'en épuiser le sens ${ }^{32}$.

Ce n'est pas que l'œuvre doive être absolument hermétique ou obscure. Elle doit au minimum faire l'impasse sur ses déterminations et laisser au lecteur un espace de "jeu" - à prendre aux deux sens du terme - qu'il ne pourra pas épuiser et qui lui laissera toute latitude pour instancier les contextes de compréhension dans lesquels l'œuvre fera le plus de sens et contribuera le plus à nourrir son esprit. Cela suppose que le texte garde une zone d'ombre, qu'il ménage une part de non-dit, de secret, ne serait-ce que dans le vouloir-dire initial qui l'a engendré. Le halo de littérarité est précisément constitué par cet appel à un dépassement du sens. Robert Scholes a formalisé cette exigence en

31 Alain-Marie Bassy, * Fablex, in Jean-Pierre de Beaumarchais et al, Dictionnaire des littératures de langue française, Paris, 1987, p. 843.

32 Pour Jean-Noël Vuamet: *On écrit pour sécréter du secret. (cité par Jacques Drillon, Traité de la ponctuation française, Gallimard, coll. *Tel *, 1991, p. 146). 
inscrivant l'ambiguïté aux six pôles du schéma de la communication proposé par Jakobson: une œuvre est littéraire à partir du moment où elle serait caractérisée par une ambiguilté sur le plan du destinateur, du destinataire, du message, du contexte, du contact ou du code ${ }^{33}$. Cela suppose évidemment aussi que le lecteur ait appris à faire jouer un texte dans des contextes variés, qu'il se soit donné des schèmes de représentation souples et multiples, capables de prendre en compte tous les niveaux d'un texte. Comme le dit Michel Picard, "l'effet littéraire n'est concevable que pour le joueur expérimenté "34.

À la limite, le simple fait, pour un tel lecteur, de décider qu'un texte est littéraire l'amènerait, après désancrage du contexte originel, à instancier ses filtres de polysémie et d'indécidabilité et, par le fait même, à y trouver le frisson esthétique caractéristique de l'effet littéraire ${ }^{35}$. Mais il ne faudrait pas pour autant reporter sur un effet de lecture la totalité du phénomène qu'est la littérarité, car cela reviendrait à oublier le socle cognitif identifié plus haut, et qui ne peut s'être engendré lui-même.

Un autre écueil à éviter serait de donner une place extrême, voire toute la place, à l'institution littéraire. Certes, celle-ci, par ses effets de légitimation, confère à des œuvres un halo de littérarité, et est à même de créer chez les lecteurs une réceptivité spéciale, facilitant l'admiration et la mise en place d'une lecture littéraire. Mais l'institution littéraire ne peut imposer durablement des œuvres qu'en tablant sur des qualités intrinsèques qui sauront réveiller chez le lecteur le sentiment de jouissance que l'expérience du langage a déjà suscitée chez lui.

Si le XVIII' siècle, comme on l'a vu à propos de l'abbé Delille, a cru pouvoir faire reposer le littéraire sur un ensemble de traits objectifs d'ordre interne (et relatifs à l'application du code rhétorique), la critique de notre époque est parfois tentée de rapporter le phénomène à des traits objectifs d'ordre externe, telle l'institution, dans laquelle il se dissout. Dans les deux cas, on fait bon marché de l'expérience de la lecture et du rapport fantasmatique au langage.

33 Robert Scholes, Semiotics and Interpretation, New Haven/London, Yale University Press, 1982.

34 Michel Picard, La lecture comme jeu. Essai sur la littérature, Paris, Minuit, 1986, p. 242.

35 Lire Umberto Eco, Lector in fabula, Paris, Grasset, 1985, p. 73-75. 
Étant toujours vécue comme le point de fuite du langage, son lieu mythique de perfection et de renouvellement, la littérature contient en elle-même son principe d'indétermination, plus affirmé maintenant que jamais, et qui interdit en même temps que puisse jamais aboutir durablement la tentative de la faire tenir dans les décrets d'une instance unique, fût-elle idéologique. 